\title{
THE NEWSROOM: USO DE UNA SERIE DE TELEVISIÓN PARA LA FORMACIÓN ÉTICA DE FUTUROS PROFESIONALES DE LA INFORMACIÓN
}

\section{The newsroom: The use of a television series for the ethical training of future information professionals}

\author{
María-Teresa Nicolás-Gavilán, Sara-Elvira Galbán-Lozano y Claudia-Fabiola \\ Ortega-Barba
}

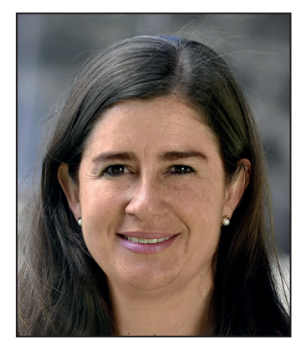

María-Teresa Nicolás-Gavilán es profesora e investigadora de la Escuela de Comunicación de la Universidad Panamericana, Campus Ciudad de México. Maestra en Ciencias Políticas y Sociales y doctora en Comunicación por la Universidad de Navarra, España; y licenciada en Derecho por la Universidad Panamericana. Es miembro del Sistema Nacional de Investigadores (SNI) en México. Sus líneas de investigación son la ética de la comunicación, los valores en las series de televisión, cobertura de conflictos y peace journalism. Es la chair del Working Group Ethics of Society and Ethics of Communication de la International Association of Media Communication Research (lamcr). Es líder de la red temática de investigación: El ADN de las series.

http://orcid.org/0000-0001-7385-8196

mnicolas@up.edu.mx

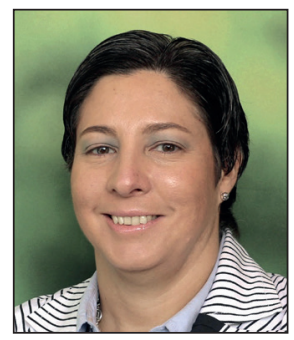

Sara-Elvira Galbán-Lozano es profesora-investigadora de la Escuela de Pedagogía de la Universidad Panamericana, Campus Ciudad de México. Doctora en Pedagogía por la Universidad de Barcelona, España. Maestra y licenciada en Pedagogía por la Universidad Panamericana. Sus líneas de investigación son: actores del proceso de enseñanza aprendizaje, práctica reflexiva y metodología de la investigación. Miembro de las redes temáticas de investigación: $E I A D N$ de las series y $L a$ enseñanza de la investigación.

http://orcid.org/0000-0002-9915-0441

sgalban@up.edu.mx

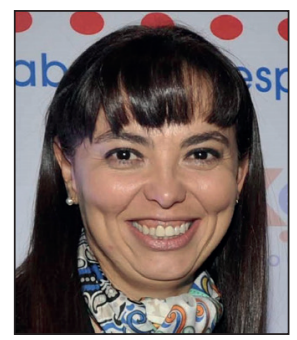

Claudia-Fabiola Ortega-Barba es profesora-investigadora en la Escuela de Pedagogía de la Universidad Panamericana. Maestra y licenciada en Ciencias de la Comunicación y doctora en Pedagogía por la Universidad Nacional Autónoma de México. Es miembro de la Sociedad Mexicana de Cómputo en la Educación y de las redes temáticas de investigación: El ADN de las series y La enseñanza de la investigación. Su línea de investigación es: mediaciones tecnológicas en procesos educativos para la innovación.

http://orcid.org/0000-0003-2709-2024

cortega@up.edu.mx

Universidad Panamericana. Campus México

Resumen

Augusto Rodin, 498. Col. Insurgentes Mixcoac. 03920 Ciudad de México, México

¿Cómo lograr que los estudiantes de comunicación integren en su vida profesional comportamientos éticos? Para responder a esta pregunta, el presente trabajo presenta el uso de las series de televisión en la docencia universitaria, como medio de aprendizaje. El artículo se configura en tres apartados: en el primero se presenta a la enseñanza universitaria como el contexto y a la ética profesional como el espacio curricular donde se desarrolla la propuesta. En el segundo se explica por qué el uso de series de televisión, además de motivar el interés de los estudiantes, facilita la adquisición de conocimientos, habilidades y actitudes éticas. En el tercer apartado se describe el uso de la serie The newsroom en el aula y algunos testimonios que evidencian la efectividad de la estrategia.

\section{Palabras clave}

Comunicación; Periodismo; Ética; Deontología; Series de televisión; Narratología; Empatía; Enseñanza; Estudiantes; Universidad; The newsroom. 


\begin{abstract}
How can we foster ethical professional behavior in communication students? In order to answer this question, this article describes how a TV series was used in university teaching as a learning tool. This article consists of three sections: first, university teaching is presented as the context and professional ethics as the curricular space where the proposal is developed; second, we show why the use of TV dramas, besides motivating student interest, facilitates the acquisition of knowledge, skills, and ethical attitudes; third, we describe the use of a TV series, The newsroom, in the classroom and some testimonies that demonstrate the effectiveness of the strategy for the understanding of the subject content.
\end{abstract}

\title{
Keywords
}

Communication; Journalism; Ethics; Deontology; TV drama; Narratology; Empathy; Education; Students; University; The Newsroom.

Nicolás-Gavilán, María-Teresa; Galbán-Lozano, Sara-Elvira; Ortega-Barba, Claudia-Fabiola (2017). "The newsroom: uso de una serie de televisión para la formación ética de futuros profesionales de la información". El profesional de la información, v. 26, n. 2, pp. 277-282.

https://doi.org/10.3145/epi.2017.mar.14

\section{Introducción: Enseñanza universitaria y enseñanza de la ética profesional}

La universidad, tal como hoy la conocemos, surgió en la Edad Media como resultado del proceso de reorganización social y cultural de la sociedad europea occidental urbana de los siglos XI y XII. La universidad aparece unida a la concepción de trabajo gremial, en donde el maestro dedicado al oficio de enseñar, era un artesano más en aquel mundo medieval de artesanos y comerciantes. La universitas es el gremio o corporación de los que enseñan y aprenden, de los maestros y discípulos dedicados al oficio de aprender los saberes (Tünnermann, 2003, pp. 21-22).

Las tendencias en educación que buscan responder a las exigencias del mundo actual, señalan que la universidad, como punta superior de la pirámide de los sistemas educativos, no tiene que replantearse sus funciones sustantivas o sus propósitos y objetivos originarios, pero sí la forma de acceder al conocimiento y las estrategias relacionadas con el proceso de aprendizaje (Martínez-Martín; Buxarrais-Estrada; Esteban-Bara, 2002; González-Tirados; González-Maura, 2007; Galbán-Lozano, 2016).

La preparación de estudiantes de comunicación para la vida profesional implica, entre otros aspectos:

- formarlos en competencias, especialmente en el uso de las tecnologías que aparecen de manera continuada; un ejemplo puede ser la reciente utilización de drones en programas informativos (Gallardo-Camacho; Lavin, 2016);

- dotarlos de un sentido de responsabilidad social para que puedan contribuir al bien común de la sociedad donde trabajarán.

La función científica de la universidad no puede restringirse en el contexto actual de crisis científica y social, a una simple tarea aséptica de generar, ser depositaria y transmitir la producción científica y tecnológica, sino que debe fomentar la formación en sólidos principios éticos de sus estudiantes.

También las empresas informativas de todo el mundo están preocupadas por el comportamiento ético de sus colaboradores y por eso han establecido por ejemplo una serie de reglas para garantizar que sus periodistas actúen con ética en el uso de redes sociales (Herrera-Damas, 2013).

El papel de la universidad en relación con la enseñanza de la ética profesional es indispensable, pues ésta crea una base intelectual, moral y social del ejercicio de la profesión, construyendo la identidad profesional. En definitiva, la formación de estudiantes de comunicación implica la adquisición de competencias propias de la profesión y la ética como forma de vida.

\section{La función científica de la universidad debe fomentar la formación en sólidos principios éticos de sus estudiantes}

La enseñanza de la ética profesional del comunicador debe considerar tres aspectos:

- comprensión de la importancia de la ética en la vida personal y profesional (Cobo-Suero, 2004, p. 271);

- bienes que protege la profesión del comunicador: verdad, libertad de expresión, derecho a la información, etc.;

respeto de los derechos de los usuarios y los clientes de los servicios profesionales, y las obligaciones de los profesionales con su gremio (Codina, 2004).

Como consecuencia de lo anterior, la incorporación de la ética profesional a los planes de estudio busca promover en los estudiantes de comunicación la reflexión sistemática sobre el servicio que prestarán a la sociedad, sus beneficiarios directos e indirectos, sus derechos y obligaciones y los posibles dilemas éticos que encontrarán en el ejercicio de la profesión (Codina, 2005; Lamberth et al., 2004). Es tal la responsabilidad social del profesional de la comunicación que no se puede pensar en él sin una formación ética.

\section{Uso de productos audiovisuales para enseñar ética profesional a estudiantes de comunicación}

Un reto de la formación universitaria es enseñar a pensar para que el alumno aprenda a descubrir la realidad, a ser experto en la aproximación al objeto de estudio desde la realidad social donde vive. De esa manera estará preparado 
para enfrentarse en cualquier momento de su vida profesional a la esencia de las cuestiones, y podrá ser flexible ante la adquisición de los nuevos saberes y quehaceres que encontrará a lo largo de su vida.

Desde su aparición se señaló que la televisión iba a ser una gran herramienta para transmitir conocimientos, ideas y experiencias (Hilliard, 1958; Linz 1958). Con el paso de los años se ha mostrado que la televisión, y especialmente los programas de entretenimiento, son un método complementario en el proceso de enseñanza-aprendizaje (Ignatieff, 1985; Vilches, 1993; Shelton, 2001; Sandoval-Escobar, 2006, White, 2008).

¿Por qué las series de televisión colaboran en el proceso de enseñanza-aprendizaje con tanto éxito? En primer lugar porque muestran relatos similares a los que ocurren en la vida real. Las historias de ficción son una forma de fomentar la imaginación moral y proveer escenarios realistas para el análisis ético (Borden, 1998). Esta misma autora sostiene que el uso de narrativas de ficción para enseñanza ética se puede integrar con las propuestas de Maclntyre (2007), quien sugiere que cada uno somos los protagonistas -de nuestra propia historia- buscando la mejor forma de vivir (Borden, 1998).

Otro factor es la empatía, un proceso multidimensional con varios niveles que pueden seguir aumentando con el tiempo (Nicolás-Gavilán et al., 2015). La televisión tiene un efecto en las prácticas sociales y culturales debido a que el público se identifica con los personajes y siente empatía hacia ellos, generando una vivencia emocional (Igartua-Perosanz; PáezRovira, 1998).

Las series de televisión se han convertido en un parámetro de referencia para las audiencias ya que tienen efectos directos sobre ellas; de hecho se ha acuñado el término narrative ethics (Phelan, 2014).

En un estudio comparado de 14 países de la Unión Europea que refleja las acciones de 50 profesores de ética periodística, se obtuvieron los resultados que se muestran en la tabla 1 (Díaz-del-Campo-Lozano, 2012).

En México y en general en Latinoamérica, no existen estudios de referencia para comparar, por lo que el presente artículo es un primer esfuerzo de aportación a la investigación. La propuesta que presentamos busca una renovación metodológica en la formación universitaria, mediante una mayor interactividad entre profesores y alumnos, a partir de un adecuado manejo de recursos y estrategias para entrar en relación con el contenido.
El uso de series en el aula busca una mayor interactividad entre profesor y alumno, a partir de una estrategia previamente pensada para entrar en relación con el contenido, en donde el uso de las series de televisión con propósitos formativos acerca al estudiante al mundo real y le permite ejercitarse en la solución de problemas y en la toma de decisiones particularmente orientadas a la formación de principios éticos. En definitiva, los mejores medios para la enseñanza y el aprendizaje son aquellos que son cercanos al estudiante.

Es tal la responsabilidad social del profesional de la comunicación que no se puede pensar en él sin una formación ética

\section{The newsroom y la práctica ética en el ejercicio de la profesión}

La enseñanza de la ética a estudiantes de comunicación busca que éstos reflexionen sobre el servicio que prestarán a la sociedad, sus derechos y obligaciones y los posibles conflictos que van a encontrar. Uno de estos conflictos se refiere a las distintas presiones o influencias que van a experimentar en la práctica profesional. Si nos centramos en la labor del periodista, un enfoque ético y realista nos lleva a cuestionarnos cómo se mantendrán los valores éticos -defensa de la verdad, derecho a la información- ante las presiones políticas, comerciales y de grupos de interés.

Dos investigadores norteamericanos, conscientes de la situación laboral de los profesionales de la información, pro-

Tabla 1. Acciones de 50 profesores de ética periodística. Resultados totales.

\begin{tabular}{|l|c|c|c|c|}
\hline & $\begin{array}{c}\text { Muy } \\
\text { frecuente }\end{array}$ & Frecuente & $\begin{array}{c}\text { Poco } \\
\text { frecuente }\end{array}$ & Nunca \\
\hline Libros y/o artículos & 58,0 & 28,0 & 8,0 & 6,0 \\
\hline Casos prácticos & 54,0 & 36,0 & 6,0 & 4,0 \\
\hline Principios deontológicos & 36,0 & 36,0 & 12,0 & 16,0 \\
\hline Ensayos y ejemplos escritos & 32,0 & 40,0 & 12,0 & 16,0 \\
\hline Discusiones en grupo & 30,0 & 28,0 & 26,0 & 16,0 \\
\hline Presentación de trabajos grupos & 28,0 & 14,0 & 24,0 & 34,0 \\
\hline Transparencias & 26,0 & 24,0 & 22,0 & 28,0 \\
\hline Presentación trabajos individuales & 26,0 & 20,0 & 20,0 & 34,0 \\
\hline Estudio de temas seleccionados & 22,0 & 36,0 & 26,0 & 16,0 \\
\hline Lecciones magistrales & 16,0 & 20,0 & 22,0 & 42,0 \\
\hline Profesionales ajenos & 12,0 & 26,0 & 36,0 & 26,0 \\
\hline Diapositivas & 12,0 & 12,0 & 24,0 & 52,0 \\
\hline Programas de televisión & 10,0 & 42,0 & 22,0 & 26,0 \\
\hline Entrevistas a profesionales & 8,0 & 14,0 & 28,0 & 50,0 \\
\hline Mesas redondas / seminarios & 6,0 & 38,0 & 18,0 & 38,0 \\
\hline Películas & 6,0 & 32,0 & 28,0 & 34.0 \\
\hline Paneles de discusión & 6,0 & 32,0 & 20,0 & 42,0 \\
\hline Role playing & 4,0 & 10,0 & 34,0 & 52,0 \\
\hline Docentes de otros departamentos & 8,0 & 42,0 & 56,0 \\
\hline & & & & \\
\hline
\end{tabular}

Fuente: Díaz-del-Campo-Lozano (2012) 
pusieron el modelo de la "jerarquía de influencias", que establece una clasificación por niveles de los factores que influyen en el periodista (Shoemaker; Reese, 2014). Los niveles son cinco:

- Individual: los autores dividen los factores de influencia en dos categorías: factores personales y profesionales.

- Rutinas profesionales: las prácticas que establece cada medio para realizar su labor y, por lo tanto, incluyen las normas, reglas y procedimientos vigentes, estructurados y profundamente enraizados en la labor de los medios. En este nivel, el editor opera como un gatekeeper.

- Nivel organizativo del medio: hace referencia a los objetivos y políticas del medio. Éste, a través de sus normas, busca conseguir sus objetivos, que muchas veces son más económicos que periodísticos.

- Elementos externos al medio como los poderes políticos y económicos: gobierno, anunciantes, departamentos de relaciones públicas, fuentes de información, grupos de presión e incluso otros medios pueden influir y, en muchos casos, tratan de influir en el frame de una noticia.

- Ideológico.

Estos aspectos claves de la teoría y práctica informativa, resultan difíciles de exponer a alumnos universitarios que nunca han ejercido el periodismo. Se presenta el reto docente de cómo hacérselos entender. Las estrategias pedagógicas únicamente argumentativas se quedan cortas, por lo que se ha optado por el uso de la ficción audiovisual, es decir, el uso de series que muestren cómo es el ejercicio profesional de un periodista.

La serie The newsroom, creada por Aaron Sorokin y emitida por la cadena $H B O$, es un producto audiovisual idóneo para tratar las cuestiones éticas a las que se enfrenta el profesional de la información. La serie nos adentra en las entrañas de la redacción del noticiero estelar de una cadena de televisión.

El elenco lo integran Emily Mortimer, John Gallagher, Jr., Alison Pill, Thomas Sadoski, Dev Patel, Olivia Munn y Sam Waterston. Sorkin -creador de El ala oeste de la Casa Blanca- desarrolló la serie desde 2009 y, para ello, observó varios programas de noticias por cable. Después de meses de negociaciones, la cadena $H B O$ ordenó un piloto en enero de 2011 y posteriormente una temporada completa en septiembre de ese mismo año. El 24 de junio de 2012 se emitió el primer capítulo.

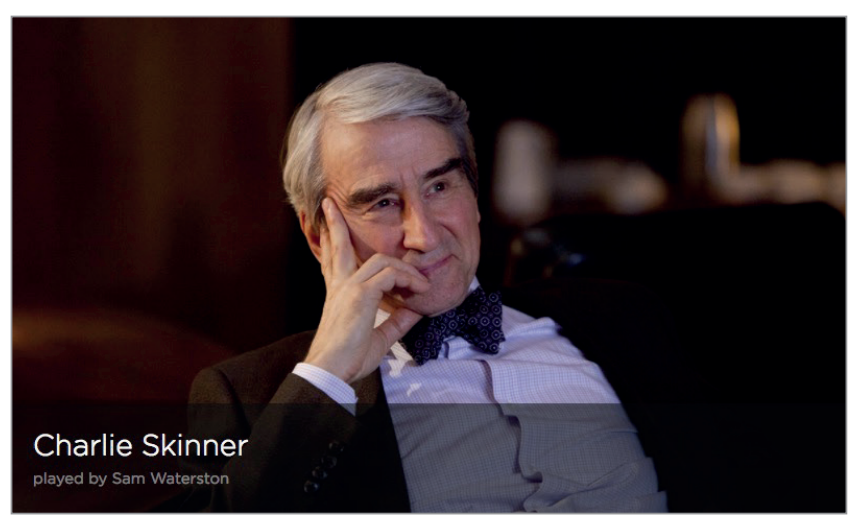

http://www.hbo.com/the-newsroom
El capítulo piloto de la serie nos presenta a sus personajes y nos introduce en el drama que se desarrolla en la redacción y foros de uno de los noticieros más populares de la televisión norteamericana. A lo largo del capítulo podemos ver al protagonista, el veterano periodista Will McAvoy, enfrentarse a varios dilemas éticos con los que no sabe cómo lidiar. Su nivel de popularidad, estrechamente ligado a su capacidad de generar dinero, se ha convertido en su principal motor y con tal de mantener esos niveles de audiencia y sueldo, su perfil como periodista y profesional en información toma un giro que se aleja de lo éticamente correcto.

McAvoy, con el afán de mantener sus ratings y el aprecio tanto de políticos como del público, decidió tener un estilo para tratar las noticias muy neutrales y hasta cierto punto cómodo. Su actitud ante el equipo de trabajo dejaba mucho que desear y ante un evento desafortunado en una conferencia dentro de una universidad, su noticiero y su perfil se ponen en riesgo. Su equipo renuncia y el jefe de la cadena manda llamar a una nueva productora, MacKenzie McHale.

MacKenzie llega con gente de su equipo y con la firme idea de crear un buen noticiero y de regresar al estilo que McAvoy tenía en sus inicios: honesto, veraz, preocupado por llevar información completa y real a su público. Le dice a McAvoy que juntos pueden crear un noticiero que sea bueno, honesto y que mantenga su nivel de popularidad.

\section{Metodología didáctica}

Esta serie se ha utilizado en la asignatura de Ética profesional, como estrategia de enseñanza/aprendizaje, con dos generaciones de estudiantes (2014/2015). Cada generación estaba integrada por más de 30 alumnos del último año de la carrera de Comunicación de la Universidad Panamericana.

La serie se proyectó en tres sesiones consecutivas. La secuencia de las sesiones se iniciaba con la presentación del contenido teórico y a continuación se proyectaba un capítulo de la serie con duración de una hora aproximadamente. Después se discutía con los alumnos el contenido del capítulo y su relación con los temas tratados, y con esto se daba por terminada la sesión. Como actividad extra los estudiantes tendrían que plasmar en un ensayo su reflexión sobre la serie y su relación con los contenidos de la clase.

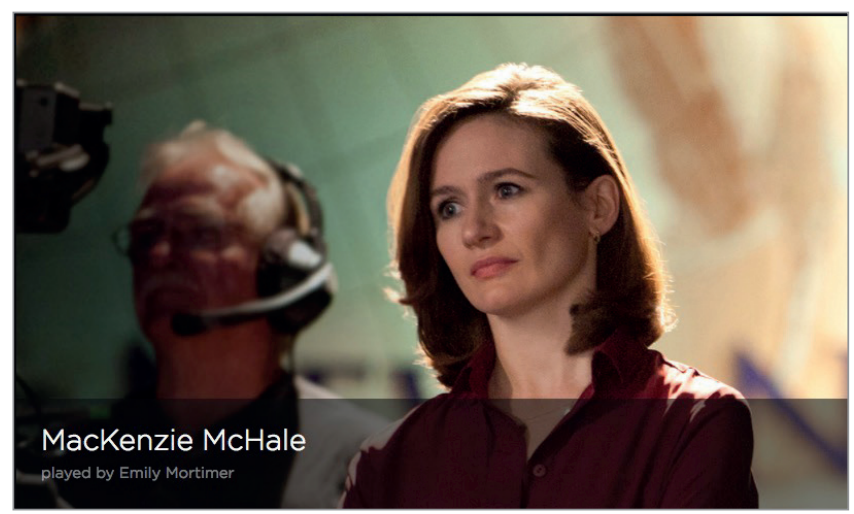

http://www.hbo.com/the-newsroom 


\section{Resultados, discusión y conclusiones}

A continuación se presentan cinco reflexiones que plasmaron los alumnos en los ensayos y que mostraban un aprendizaje significativo.

"The newsroom sirve para cuestionarnos y discutir la realidad de la información y las noticias, cómo éstas se han transformado en revistas informativas que no muestran ni dejan nada en realidad" (alumno 1 ).

Además de hacer reflexionar a los alumnos, la visualización de la serie les permitía entender la realidad del trabajo de un periodista. Así lo refleja una alumna:

"Lo que más me gustó del capítulo es la rapidez en los diálogos, la ironía y el sarcasmo que maneja. La adrenalina de la sala de prensa y el manejo de la tensión me parece extremadamente interesante en el momento en que se analiza el por qué se transmite la información que se transmite" (alumna 5).

Las dos reflexiones siguientes hechas por alumnos correspondían a la proyección del capítulo piloto de la serie y se centra en una reflexión más global sobre la profesión. Además muestran como el componente de "disfrute" de la serie fortalece la experiencia de aprendizaje.

"En la serie se presentan muchos dilemas éticos, cada uno de los personajes tiene que lidiar con lo que se enfrenta y lo hace de manera distinta.

Will Mcavoy se aleja de la superficialidad de la importancia de los ratings y toma el riesgo de hacer televisión de calidad para los televidentes.

MacKenzie sigue sus convicciones, es una buena productora que busca decir la verdad sin importar lo que los demás piensen, tiene claro sus proyectos y sus convicciones.

Charlie: este personaje se encuentra en una posición delicada ya que defiende sus creencias y defiende al programa aunque éste vaya en contra de los intereses económicos de la empresa. Y dice lo que piensa" (alumna 2).

"Desde el punto de vista ético me parece que los nuevos principios sobre los cuales se hace el programa de televisión son correctos: se busca la relevancia, la verdad y la objetividad de los hechos para presentarlos a la población. Como menciona Charlie, Will se ha convertido en un "abogado" para el pueblo americano y busca dar una versión verídica y sin intereses comerciales hacia la ciudadanía. Es aquí que llegamos a dos puntos muy importantes de analizar. En primer lugar, un canal de noticias sigue perteneciendo a una cadena que tiene intereses políticos y económicos. Es esta situación la que causa que los altos mandos no estén conformes con los nuevos juicios que Will (el presentador) transmite al aire, dejando "mal parados" a políticos que pueden injerirse en lo que respecta a los medios de comunicación en los años venideros. El interés y la preocupación de los directivos me parece normal. Sin embargo, no así se puede pasar por alto la parte ética de la situación en la que un medio no puede venderse con tal de seguir en el negocio, el primer interés debe estar siempre en el informar. En segundo lugar, tenemos al personaje de Will como tal.

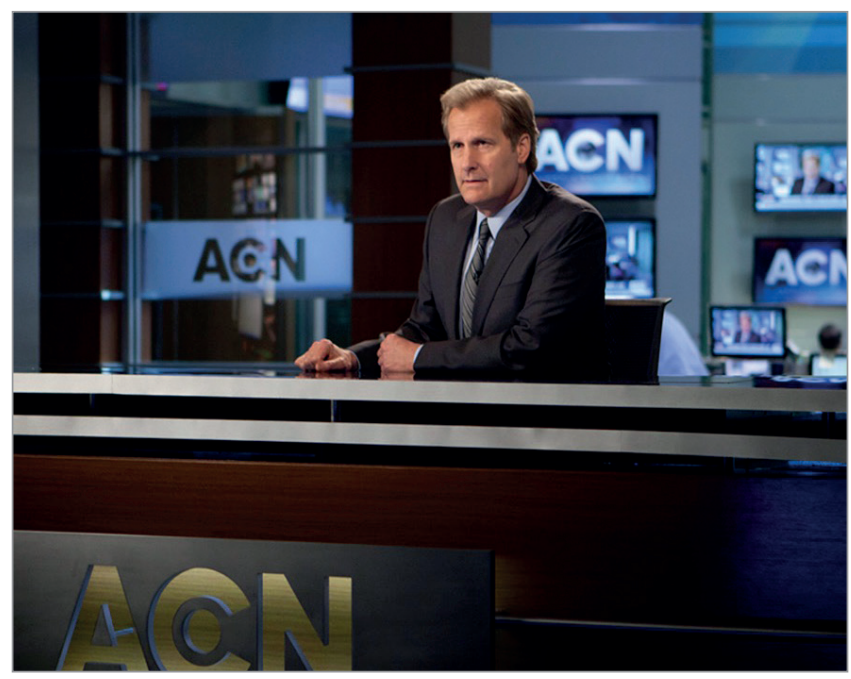

McAvoy. http://www.hbo.com/the-newsroom

Su personalidad y sus antecedentes parecen justificar la manera con la que ataca a sus entrevistados a pesar de que en ocasiones es demasiado duro. No me parece mal que ejerza este tipo de acciones, pero es importante que igual como es con la "oposición" debe serlo con el partido que le parece más correcto" (alumno 3).

Estos dos comentarios de los alumnos muestran que fue un acierto de los guionistas establecer como protagonistas de las historias a dos personajes con características tan distintas: edad, sexo, origen y visiones de la profesión del informador. Estos contrastes, además de permitir el conflicto que mueve la historia, permiten que los alumnos, con sus distintas personalidades se identifiquen con cada uno de los protagonistas y hagan suyos los conflictos vividos por los mismos. Esta empatía logra poner en "situación real" a los estudiantes con relación con los conflictos y dilemas éticos que implica ejercer el periodismo.

El siguiente comentario nos permitió comprobar que las enseñanzas éticas de la serie trascienden a las circunstancias particulares que se narran en la serie, que se sitúa en Estados Unidos, ya que la alumna la extrapola a la situación de su país.

"Los periodistas tienen la responsabilidad de tomar decisiones importantes en cuestión de segundos, decir la verdad los convertirá en profesionales con una ética y moral que sean ejemplo a seguir. Y así, si cada persona que trabajara en los medios de comunicación lo fuera, se tendría un país en donde todos coincidan y se comunicarían noticias más íntegras. La serie me hizo reflexionar sobre mi país, ya que en México existen profesionales con gran ética y moral pero la mayoría se ha dejado llevar por el dinero y la corrupción que se encuentra a su alrededor. Si cada uno de nosotros, los que seguimos estudiando, tuviéramos una formación ética y profesional el mundo que nuestros padres nos están dejando estaría mejor informado" (alumna 4).

Con los testimonios anteriores se puede visualizar que el uso de esta serie como estrategia formativa ha resultado útil en el tratamiento de ciertos temas de la asignatura de Ética profesional para periodistas. La estrategia es eminen- 
temente práctica, con ella se pretende que los estudiantes interioricen críticamente valores, desarrollen actitudes responsables y se acostumbren a pensar como profesionales de la comunicación, aplicando conceptos y conociendo perspectivas del campo de trabajo. Lo anterior se logra a partir del análisis, discusión, debate y argumentación de casos mostrados en la serie.

La utilización de las series de televisión enriquece la labor docente del aula, permitiendo que los estudiantes universitarios se adentren en las salas de redacción de los noticiarios de manera virtual, mediante la ficción audiovisual, logrando la vivencia que todavía no han experimentado. Con esto se trata de acercar el objeto de estudio al mundo vital del estudiante.

A partir de los testimonios recogidos vislumbramos que el uso de series puede ser útil en la formación ética de los futuros profesionales de la información. Sin embargo, para la elaboración de un modelo pedagógico replicable, se necesita la realización de investigaciones que arrojen resultados generalizables, lo cual se pretende hacer en futuros trabajos.

\section{Bibliografía}

Borden, Sandra (1998). "Avoiding the pitfalls of case studies". Journal of mass media ethics, v. 13, n. 1, pp. 5-13. https://doi.org/10.1207/s15327728jmme1301_1

Cobo-Suero, Juan-Manuel (2004). “Universidad y ética profesional". Teoría de la educación. Revista interuniversitaria, v. 15 , pp. $258-276$.

http://gredos.usal.es/jspui/bitstream/10366/71945/1/ Universidad_y_etica_profesional.pdf

Codina, Mónica (ed.) (2004). De la ética desprotegida. Ensayos sobre la deontología de la comunicación. España: Eunsa. ISBN: 9788431322106

Díaz-del-Campo-Lozano, Jesús (2012). “Técnicas pedagógicas básicas para la enseñanza de la ética periodística". Revista científica electrónica de educación y comunicación en la sociedad del conocimiento, v. 2, n. 4, pp. 303-324.

http://eticanet.org/revista/index.php/eticanet/article/ view/13

Galbán-Lozano, Sara-Elvira (2016). Hacia una enseñanza reflexiva. México: Trillas. ISBN: 9786071725561

Gallardo-Camacho, Jorge; Lavín, Eva (2016) “Uso de drones con fines informativos en empresas de televisión en España". El profesional de la información, v. 25, n. 2, pp. 217-225. http://dx.doi.org/10.3145/epi.2016.mar.08

González-Tirados, Rosa-María; González-Maura, Viviana (2007). "Diagnóstico de necesidades y estrategias de formación docente en la universidad". Revista iberoamericana de educación, v. 6, n. 43, pp. 1-14.

http://rieoei.org/deloslectores/1889Maura.pdf

Herrera-Damas, Susana (2013) "Indicaciones recurrentes en las normativas para el uso periodístico de las redes sociales". El profesional de la información, v. 22, n. 1, pp. 46-53. http://dx.doi.org/10.3145/epi.2013.ene.06
Hilliard, Robert (1958). "Television and education". The journal of higher education, v. 29, n. 8, pp. 431-470.

Igartua-Perosanz, Juan-José; Páez-Rovira, Darío (1998). “Validez y fiabilidad de una escala de empatía e identificación con los personajes". Psicothema, v. 10, n. 2, pp. 423-436. http://www.psicothema.com/pdf/175.pdf

Ignatieff, Michael (1985). "Is nothing sacred? The ethics of television”. Daedalus, v. 4, n. 114, pp. 57-78.

Lambeth, Edmund B.; Christians, Cliford G.; Fleming, Kenneth; Lee, Seow-Ting (2004) "Media ethics teaching in century 21: Progress, problems, and phallenges". Journalism \& mass communication educator, v. 59, n. 3. pp. 239-258. https://doi.org/10.1177/107769580405900304

Linz, C. C. (1958). "Television and education". The Australian quarterly, v. 30, n. 3, pp. 36-45.

Maclntyre, Alasdair (2007). After virtue: A study in moral theory. London: Duckworth. ISBN: 9780268035044

Martínez-Martín, Miquel; Buxarrais-Estrada, María-Rosa; Esteban-Bara, Francisco (2002). "La universidad como espacio de aprendizaje ético". Revista iberoamericana de educación, n. 29, pp. 17-42

http://rieoei.org/rie29a01.htm

Nicolás-Gavilán, María-Teresa; Quintanilla-Jiménez, Carmen; Padilla-Lavín, María-de-los-Ángeles; Vargas-Zamorano, Perla-Paola (2015). "A woman of the 60's caught in a contemporary TV series: Claire Dunphy a housewife in a Modern family". Communication \& social change, v. 3, n. 1, pp. 19-47. http://dx.doi.org/10.17583/csc.2015.1774

Phelan, James (2014). “Narrative ethics”. En: Hühn, Peter; Meister, Jan C.; Pier, John; Schmid, Wolf (eds.). The living handbook of narratology. Hamburg: Hamburg University. http://www.Ihn.uni-hamburg.de

Sandoval-Escobar, Marithza (2006). "Los efectos de la televisión sobre el comportamiento de las audiencias jóvenes desde la perspectiva de la convergencia y de las prácticas culturales". Universitas psychologica, v. 5, n. 2, pp. 205-222. http://www.redalyc.org/articulo.oa? id $=64750202$

Shelton, Courtney (2001). "Must see TV: The timelessness of television as a teaching tool". Journal of management education, v. 25, n. 6, pp. 631-647.

https://doi.org/10.1177/105256290102500603

Shoemaker, Pamela J.; Reese, Stephen D. (2014). Mediating the message in the $21^{\text {st }}$ Century: A media sociology perspective. USA: Routledge. ISBN: 9780415989145

Tünnermann, Carlos (2003). La universidad latinoamericana ante los retos del siglo XXI. México: UDUA. ISBN: 978 9686802221

Vilches, Lorenzo (1993). La televisión, los efectos del bien y del mal. Barcelona: Paidós. ISBN: 9788475099101

White, Gladys (2008). "Capturing the ethics education value of television medical dramas". The American journal of bioethics, v. 12, n. 8, pp. 3-14.

http://dx.doi.org/10.1080/15265160802568782 